$54^{\text {ème }}$ Congrès de la SFMBCB, 02008 (2011)

DOI: $10.1051 / \mathrm{sfmbcb} / 20115402008$

(C) Owned by the authors, published by EDP Sciences, 2011

\title{
A propos d'un cas de B-thalassémie : nouvelles implications de cette pathologie et de ses traitements en chirurgie buccale
}

\author{
Millot $\mathrm{S}^{\mathbf{1 , 2}}$, Woimant $\mathrm{H}^{\mathbf{3}}$, Ejeil AL $\mathbf{L}^{\mathbf{1}}$, Charrier $\mathrm{JL}^{\mathbf{1}}$ \\ ${ }^{1}$ Faculté de Chirurgie dentaire, Université Paris Descartes, Paris, France \\ ${ }^{2}$ INSERM U773, "Fer et synthèse d'hème ", Paris Diderot site Bichat, Paris, France \\ ${ }^{3}$ Service de Stomatologie, Hôpital Saint Antoine, Paris, France \\ sarahmillot@yahoo.fr
}

Les thalassémies, hémoglobinopathies génétiques très répandues (270 millions de porteurs dans le monde), sont dues à un défaut de la production de chaînesa ou $\beta$ de la globine (Rund et al. 2005). Ces syndromes couvrent des phénotypes variés, le plus sévère étant la $\beta$-thalassémie majeure homozygote, se caractérisant par une érythropoïèse inefficace et une hémolyse. Il en résulte une anémie sévère (hémoglobine à $7 \mathrm{~g} \cdot \mathrm{dL}^{-1}$ ) qui stimule la production d'érythropoïétine entraînant, dans la moelle osseuse, une prolifération massive des précurseurs des globules rouges à l'origine, entre autres, de déformations osseuses touchant notamment de la face. Misch et al. (1998) ont décrit des déformations du maxillaire et de la mandibule avec diminution de l'épaisseur des corticales, oblitérations plus ou moins complètes des sinus maxillaires et des troubles de l'articulé dentaire. Malgré un traitement complexe (transfusions sanguines une fois par mois...) et lourd, la qualité de vie des patients s'est améliorée ces dernières années, et leur espérance de vie a été doublée. Ces avancées s'accompagnent de complications pour la plupart liées à la surcharge en fer : atteintes du foie et des glandes endocrines, ostéoporose, diabète, insuffisance et /ou arythmie cardiaques (responsable de $70 \%$ de décès dans la thalassémie majeure) (Cunningham et al. 2004, Borgna et al. 2005).

Une patiente de 28 ans est adressée dans le Service de Stomatologie par le Service des Maladies infectieuses pour un syndrome inflammatoire avec hyperthermie $\left(40.2^{\circ} \mathrm{C}\right)$, douleurs et une adénopathie sous-angulo-maxillaire gauche. Le passé médical révèle une $\beta$-thalassémie majeure, une splénectomie totale, une hémochromatose posttransfusionnelle traitée par Ferriprox, arrêté depuis par la patiente. Les examens biologiques sont les suivants : plaquettes $931000 \mathrm{~mm}^{-3}$, ASAT 133 UI.L ${ }^{-1}$, hémoglobine $7.5 \mathrm{~g} . \mathrm{dL}^{-1}$, leucocytes $26.2610^{9} . \mathrm{L}^{-1}$, CRP 31, ferritine $11407 \mathrm{ng} . \mathrm{mL}^{-1}$. L'examen clinique confirme l'adénopathie et montre une tuméfaction intra-buccale en regard de 37. Les examens radiographiques révèlent une image radioclaire en rapport avec 37, qui a été extraite sous couverture antibiotique. Une semaine plus tard, les anomalies biologiques persistent. La conclusion médicale est la suivante: « foyers infectieux d'origine dentaire chez une patiente à haut risque infectieux ». Il a fallu attendre 15 jours et deux transfusions sanguines pour retrouver un bilan biologique normal.

Plusieurs complications de cette pathologie interfèrent avec notre pratique :1) susceptibilité à l'infection et surcharge en fer due aux transfusions sanguines, chacune d'elle augmentant la quantité de fer ; 2) maladie osseuse : elle est multifactorielle et l'utilisation de bisphosphonates par voie intra-veineuse fait l'objet d'études, ils constituent actuellement le traitement de choix même chez les jeunes patients (Wonke et al. 1998, Voskaridou et al. 2001) ; 3) hypercoagubilité : la fréquence des accidents thrombo-emboliques chez les patients jeunes, est bien supérieure à la moyenne (Eldor et al. 2002). Ce cas clinique, plus biologique que chirurgical, met en évidence la complexité de cette pathologie et l'importance de l'éradication des foyers infectieux bucco-dentaires. Si actuellement, grâce aux traitements, les déformations osseuses sont moins fréquentes, d'autres complications surviennent qui doivent attirer notre attention: chirurgie et bisphosphonates, risque infectieux, paramètres biologiques à surveiller.

Les thalassémies comportent de nombreuses formes cliniques - le cas rapporté était une forme majeure connue - dont on doit connaître le traitement et les complications. 